\title{
Exploring Journalism and Computer Science Student Collaboration
}

\author{
A Norwegian case study
}

\author{
Elsebeth Frey ${ }^{\mathrm{I}}$, Ragnhild K. Olsen ${ }^{\mathrm{I}}$ \& G. Anthony Giannoumis ${ }^{\mathrm{II}}$
}

${ }^{1}$ Department of Journalism and Media Studies, Oslo Metropolitan University, Norway.

II Department of Computer Science, Oslo Metropolitan University, Norway.

\begin{abstract}
The digitalization of journalism has resulted in an increased overlap between technology and journalism in the newsroom. This development has profound implications for journalism education. The present study investigates a team-based experiential learning project between journalism and computer science students in a digital feature journalism course. Using the concept of trading zones as our analytical lens, we explore the students' thoughts and opinions regarding professional roles and boundaries as well as areas of tension and spaces of mutual understanding in the collaborative context. Using mixed methods and data from questionnaires, observations and semi-structured interviews, the study demonstrates how trading zones between journalism and computer science students varied from homogenous collaboration to heterogeneous coercion, with diverse experiences of collaboration, coordination and collapse.
\end{abstract}

Keywords: trading zones, technology development, journalism education, collaboration, digitalization

\section{Introduction}

The digitalization of journalism and convergence of media platforms has resulted in an increased overlap between technology and journalism. From a professional perspective, this means that technology developers have begun working as technology developerjournalists and computer science engineers have shown a growing interest in journalism (Lewis \& Usher, 2014). Leading voices in the field of journalism have also pushed for journalists to embrace computer science (Lewis \& Usher, 2016) and pointed out the profound implications of digitalization for journalism education (Hultén \& Edwardsson, 2017).

Frey, E., Olsen, R. K. \& Giannoumis, G. A. (2019). Exploring journalism and computer science student collaboration: A Norwegian case study in Nordicom Review, 40(2): 129-142. doi:10.2478/ nor-2019-0037. 
In light of the increased calls for experiential learning opportunities in journalism and for academic research into such opportunities (Parks, 2015), this article presents a small-scale study that investigates cooperation between journalism and computer science students in a joint development project offered at Oslo Metropolitan University. ${ }^{1}$ Drawing on the work of Galison (1997), Collins and colleagues (2007) and Lewis and Usher (2016), we apply the concept of the trading zone to analyse the complex dynamics and interconnections that may occur in an interdisciplinary, educational project.

Our overarching question is, 'How can journalism and computer science students collaborate in the production of digital feature journalism catering to specific user groups?' Following this, we consider three research questions:

RQ1 What are students' perceptions of their roles and responsibilities in the project?

RQ2 To what extent do the students find common ground in the project?

RQ3 What are the students' perspectives on users' needs?

The article proceeds with a literature review presenting relevant research on professional cultures and identities, trading zones and user orientation in the context of journalism and computer science. ${ }^{2}$ Following this, our mixed methods approach is described, after which the findings and their implications are presented and discussed.

\section{Literature review}

\section{Identities and professional culture}

Anderson and colleagues (2010) investigate how engineers' work identity centres on their ability to solve technical problems, to be team players and to be life-long learners. Their results show that communication and collaboration are important skills in interdisciplinary teams. Personal contributions are part of the archetypal engineer, who solves problems for the team, the organization, the client and/or the end user. Boden and Avram (2009) reveal a strong reliance in software companies on what they call knowledge brokers - that is, people who create bridges in collaboration and coordination, often due to personal inclinations and partly due to formal roles.

The main occupation of journalists is keeping the public informed by gathering and checking facts and presenting stories. Traditionally, this has been a one-way relationship, where journalists serve as gatekeepers who use their own perception of newsworthiness to select and present stories for an audience. While Wolf and Schnauber (2015) note that the digitalization of journalism has substantially affected the way journalists work, Reese (2016: 823) concludes that 'journalistic structures and routines are, perhaps surprisingly, robust' (see also Lewis \& Usher, 2014). Still, as the necessity for digital skills and tools has increased, 'the values of technology culture have become linked with journalistic practice' (Reese, 2016: 819).

Lewis and Westlund (2015) investigate the evolving relationship between journalists and end users and argue that journalists perceive them as passive recipients of news. In a similar vein, Boyles and Meyer (2016) maintain that journalists largely perceive themselves as responsible for educating and informing the audience.

In the relations between journalists and audiences, technology serves as an indispensable mediator in which technology developers are important stakeholders. While 
technology developers' identities are connected to teamwork and problem-solving in order to meet client needs, journalists' identities are tied to an independent and critical position towards everyone and everything (sources, documents, facts, etc.).

Technology developers characterize users in relation to the actions or activities associated with the use of technology, the outcomes of those actions or activities and the relationship between a user's intentions, actions and results, along with the interpretation of those results (Norman, 2001; Norman \& Draper, 1986). Research in universal design and technology accessibility examines the barriers that people - especially those with disabilities - experience using technology (Aizpurua et al., 2016). Iwarsson and Ståhl (2003: 61) argue that universal design is, first and foremost, about usability and "design for all".

In a small-scale study on the Norwegian Broadcasting Corporation (NRK), Nordli (2016) finds that NRK's publication system does not meet the national standards for universal design. He shows that 'the majority of editorial employees are unfamiliar with universal design as a concept, [and] that the majority does not take any measures towards universal design or increased accessibility when creating content' (Nordli, 2016: 75). However, journalists take steps to improve the readability of their texts, such as making their language simpler and easier to understand. Gyori and Charles (2018) find that journalism students struggle with user testing. They suggest that the best practices for journalists are to think and act like web designers: first through a three-stage testing process, second by a hieratic design that hooks the audience and then uses orientation to give them 'a clear sense of what matters and how much' (Gyori \& Charles, 2018: 213 ), third by sharing control through affordance authoring and last by consciously curating content.

\section{Trading zones}

When studying how different professional groups work together, the distinctness of their identities and the dynamics of mutual tension and interconnection, Peter Galison (1997) uses the concept of trading zone: 'the site - partly symbolic and partly spatial at which the local coordination between beliefs and action takes place' (Galison, 1997: 784). Despite their differences, distinct groups do exchange cultural knowledge in a trading zone, where both groups may 'impose constraints on the nature of exchange' (Galison, 1997: 806). They coordinate their different approaches without losing their distinctiveness, trading back and forth while preserving traditions in the collaborative process and working towards a shared goal.

When investigating the intersection of journalism and technology, Lewis and Usher (2016) use Galison's concept of trading zones, finding that journalists and technology developers experienced challenges in establishing a common language for translating their abstract aims into action. The groups differed in their familiarity with technical jargon and their focus on short-term content development versus long-term software development. While the trading zones helped create common spaces for a shared language, object and cause, 'the trading zone [also] reinforced particular differences' (Lewis \& Usher, 2016: 10).

Collins and colleagues (2007: 658) define trading zones as 'locations in which communities with a deep problem of communication manage to communicate'. These 
authors go on to describe four basic types of trading zones that lie on a spectrum from collaboration to coercion and homogeneous to heterogeneous. The first is the interlanguage trading zone, which is homogeneous and collaborative and involves mutual agreement to exchange and develop new tools and a common language. The enforced trading zone is second; it is heterogeneous and coercive and lacks a culture of sharing. The third type, dubbed a subversive trading zone, is coercive and homogeneous. Here, components from both parties are brought to the trade, and the two different languages or cultures are 'on their way to being replaced by one' (Collins et al., 2007: 660) as the imposing culture gains hegemony. Finally, in the fractionated trading zone, which is heterogeneous and collaborative, fractions of cultures are exchanged.

Research into the challenges and factors at play when journalists and programmers cooperate indicates that knowledge exchange and collaboration generate new insights (Kavanagh \& Cokley, 2011; Newell, 2013; Weber \& Rall, 2012). Although the two student groups in Kavanagh and Cokley's project 'retained their separate identities' (2011: 18 ), the students received an awareness of and identified 'the contribution of the other students' (2011: 17). Hultén and Edwardsson (2017) find challenges like communication between disciplines, finding common ground and obstacles when integrating storytelling and engineering design. However, their students found the collaboration to be helpful overall and 'reported an awareness of the need to communicate across disciplines' (2017: 13). In a similar vein, Angus and Doherty (2015) state that despite many challenges, interdisciplinarity was achieved in their student project. Weber and Rall (2012) point out that the key element to better interdisciplinary production is the attitude that every 'team member acts as a journalist' (Weber \& Rall, 2012: 354).

\section{Our case:}

\section{Journalism meets computer science at Oslo Metropolitan University}

The present study is based on a collaborative project between Norwegian journalism and computer science students at Oslo Metropolitan University during the autumn of 2016. The overall learning goal of the projects was to get the students to work together and acquire interdisciplinary experience and skills through practice. Prior to the project, there had been no formal cooperation between students or teaching staff from the two departments. The project was planned as team-based learning aimed to boost learning effectiveness by creating a student-centred, self-motivated and cooperative learning environment (see Han \& Newell, 2014). Active student participation was encouraged, as this aligned with the teachers' overall ambition to observe and analyse interdisciplinary collaboration in practice-based learning, in which classroom activities should be as similar to the real world as possible (see Pain et al., 2016).

The project was organized around four workshops with fifteen journalism students in their second year of their bachelor studies and 18 first-year computer science bachelors students following a course in web design that focused on user-centred design. In the first workshop, the students were primed for the project through a joint lecture on user needs and implications for the work of both journalists and computer scientists. The project was integrated into the mandatory portion of the journalism students' feature reporting course, while the computer science students voluntarily joined the project.

The student groups were divided into seven cross-disciplinary teams chosen at ran- 
dom by the course instructors. Each group was assigned the task of developing an online multimedia feature story. The students were asked to focus on users in their production, but there were no other guidelines on how to carry out the assignment or definition of roles for team members. While no specific learning materials were used to elicit reflections on the students' roles and responsibilities, the methods detailed below prompted students to consider their roles and responsibilities as they worked together.

\section{Methodology}

Based on the analytical framework of trading zones, the present study explored the cooperation of journalism and computer science students along the dimensions of heterogeneity versus homogeneity and collaboration versus coercion. We used a combination of qualitative methods in a mixed methods research design, which was important given the small scale of the project.

At the outset, we invited all 33 students to fill out an online questionnaire with openended questions regarding their professional positions and expectations about the roles and responsibilities of journalists and technology developers. Our aim was to obtain a broad overview of their different perceptions in this regard. To capture potential changes in perceptions of roles and responsibilities and their experiences of working together, we also distributed a questionnaire at the end of the project. In this second questionnaire, participants were encouraged to reflect on their roles in the project and the division of labour.

We received 23 completed questionnaires in the first round $(15$ computer science and 8 journalism students) and 24 in the second (13 computer science and 11 journalism students). The questionnaire data provided the basis for exploring heterogeneity and homogeneity in the groups and capturing participants' experience of cooperation as coercive or collaborative.

To obtain a deeper understanding of their views, we conducted in-depth group interviews with the students after the assignment was completed. These semi-structured interviews covered experiences with cross-disciplinary collaboration, students' roles and functions in the group, approaches to the specific journalistic assignment and the process of developing the group website. Here, we asked how they worked during the project, if anyone took on more responsibilities than others and if they faced any challenges, such as issues around different concepts. As participants were able to reflect and comment on how they defined various concepts and one another's views and roles, these interviews provided a basis for exploring social interactions (see also Gentikow, 2005).

Four of the seven interdisciplinary groups were interviewed. The groups were purposively selected to represent different group compositions and collaborative approaches observed during the workshops. To capture both individual and collective experiences, we interviewed the students in groups of two or three. In total, we conducted 10 interviews with 18 participants, (eight journalism students and ten computer science students; six women and 12 men). To avoid cross-disciplinary misinterpretations, we conducted separate interviews with journalism students and computer science students. The interviews were recorded and transcribed. When referring to respondents in the questionnaires, we use each student's future profession and questionnaire number. When referring to the interviewees, we also provide a number for the group. All translations from Norwegian are by the authors. 
The third part of our methodology was individual observations carried out by the teachers during the workshops. Our observations and reflections were discussed during follow-up meetings. By sharing thoughts and experiences from the workshops, we developed a basis for analysing and interpreting data from the questionnaires and the in-depth interviews according to the trading zone dimensions.

The analysis was carried out at research workshops where the data was categorized and discussed, as were our roles as teachers and researchers in the project. Lees (2001) refers to research on one's own profession as reflexive action research, pointing out that this approach is widely used in education research and arguing that 'continuous professional development is itself a form of reflexive action research' (Lees, 2001: 133). However, there are some challenges with this approach. The researchers' role as teachers may, for example, have affected the object under study, in this case the students' collaborative work and their reflections on what they did. Furthermore, the researchers' opinions, prejudices and other biases as teachers may have affected their interpretation of the data. One step taken in the present study to reduce this risk was to establish some distance between students and researchers-teachers in the interview situation; journalism and computers science teachers did not interview their own students. In addition, the research design and data analysis were overseen by a teacher who was not involved in either course.

\section{Heterogeneous roles and responsibilities}

Answering RQ1 on the students' perceptions of their roles and responsibilities in the project, data from the initial questionnaire showed that the journalism and computer science students regarded themselves as notably different, with separate roles and responsibilities. The groups were thus defined as heterogeneous. We found that journalism and computer science students agreed on their respective roles and responsibilities; both groups described how computer science students' responsibilities pertained to the design, form and functionality of the webpage: 'A programmer is someone who writes computer software. He [sic] can take new and innovative ideas and make them into programs, applications or websites' (technology developer, questionnaire 1). Both our observations of how the students organized their work and the data from the interviews confirmed computer science students' roles as suppliers of technological solutions, not content. As to journalists' roles and responsibilities, the data demonstrated an equally distinct understanding of journalists as a separate group. The first questionnaire showed that the students agreed that journalists' work was to gather information and present stories to an audience. In both groups, we found respondents referring to the democratic role of the press as an important part of journalists' roles and responsibilities. Throughout the project we observed that the students were sure of their own future professional roles in either journalism or technology development.

Regarding the students' expectations when starting our project, we found that the journalism students had especially high expectations concerning the computer science students' contribution to the design of the digital solution and the development of the interface to meet end users' needs. This could be interpreted as journalism students' having enormous faith in computer science students or as relying on them to do the hard work, which would magically lead to new insights that the journalists could use: technology developers should prevent technology from being a barrier and thus intro- 
duce journalists to another language and an exchange of a different professional culture (journalist, questionnaire 1).

Like Hultén and Edwardsson (2017), we found that some journalism students expected to take on a broader responsibility in the project, contributing not only to content but also to the technical solution and programming. They expected to learn another professional language and expand their skills to include design, function and formatting. Gyori and Charles (2018) state that such ambitious ideas may result from the students' lack of understanding of what is possible in design and programming. Furthermore, Kavanagh and Cokley (2011) find that high expectations contributed to later dissatisfaction.

Although we agree with Gyori and Charles (2018), we feel that the issue of expectations also involves an overlap in interests that could increase homogeneity between the groups. However, this interest in programming did not apply to all our journalism students, and our observations and interviews showed that the journalism students had not engaged in coding or programming, focusing instead on content creation. This was in line with the computer science students' expectations, which clearly indicated a heterogeneous view of the two vocational groups. In both the first questionnaire and the interviews, they described how they expected journalists to contribute content. They indicated the need to coordinate different approaches while treating the journalism students as they would any other client, and they did not express any desire to learn the cultural values or norms of journalism.

\section{Integrating experiences}

This section deals largely with how the students worked together in the production of digital feature journalism, and as such considers the first two research questions (i.e., what are students' perceptions of their roles and responsibilities in the project [RQ1], and to what extent they found common ground in the project [RQ2]). In the end-of-project questionnaire, we found that both journalism and computer science students described a clear division of responsibilities. Overall, after trading back and forth, the students saw the two professional groups as having heterogeneous tasks but still cooperating (see also Galison, 1997). Essentially, journalism students created the content while computer science students developed the webpage. That said, we observed that the level of collaboration differed significantly from one team to another (see also Angus \& Doherty, 2015; Gyori \& Charles, 2018; Hultén \& Edwardsson, 2017).

Observations on the progress of their joint work, the way they worked and the tone in which they spoke to each other all indicated the degree to which they found common ground. In addition, the questionnaire and the interviews showed different levels of cooperation along the axis of collaboration and coercion. Some groups interacted a lot, while others worked independently from one another: '[We were] very separated; we produced all the written and visual material, and the others did mostly work on the webpage. But we communicated a lot through Facebook' (journalist, questionnaire 2 ). However, interviewees in group 4 described their working process as natural and dynamic, with significant amounts of dialogue, trust, listening and sharing knowledge (interviewees, group 4). Likewise, one group worked together all the way from brainstorming the idea to achieving the final result, and this group also found common ground 
(interviewee, group 3). During the process, we observed how a shared language emerged (cf. Angus \& Doherty, 2015).

As for group 1, the students in this group also talked about professional trust between technology developers and journalists. However, the computer science students in this group looked at the journalism students as clients who were assigning them a task. This notion was confirmed by the journalism students (interviewee, group 1). This tied in with the follow-up questionnaire, which indicated that technology developers had been instructed by the journalists. One student stated, 'The journalist [as client] instructed us in what they wanted, whereas we coded' (technology developer, questionnaire 2). So, more than working side by side, they combined 'their areas of expertise customized for the requirements of the specific project' (Weber \& Rall, 2012: 351).

In one group, the interviews showed that collaboration was almost non-existent. This sense of coercion was confirmed by our observations during the workshops. We noted that one or two journalism students were almost always absent; when present, they showed little enthusiasm. Furthermore, the computer science students did not receive feedback from the journalism students on the finished webpage (interviewees, group 2). This behaviour could be due to having difficulties working outside the box (see Kavanagh \& Cokley, 2011), a lack of engagement or a wish to find "'easy" or "easier" ways out' (Gyori \& Chares, 2018: 211).

Other groups, however, rose to the challenge. Some students described how solutions were discussed and developed collaboratively as part of a truly joint effort. Others stated that the webpage was developed as a result of journalists' instructions; by contrast, it was developed in another group despite a lack of engagement from the journalism students.

As to the challenges experienced in the project, the students all said they would have liked to have more time (see also Hultén \& Edwardsson, 2017), and late delivery of content from the journalism students was a common theme reported by both computer science and journalism students. A couple of journalism students mentioned the technology developers' competence and a lack of information about project objectives as challenges. This was confirmed by observations from the workshops. In one group cooperation was so difficult - due to the misalignment of expectations between journalism and computer science students - that teachers had to intervene and mediate an open conflict. These experiences were radically different from the harmonious collaboration observed in and described by other groups.

Taking the interviews into account, we found that most journalism students praised the competence of the programmers (interviewees, groups 3 and 4) and acknowledged the computer science students' competence as distinct from the journalism students (interviewees, group 1). In the follow-up questionnaire, though, a few students referred to a lack of journalistic understanding among programmers. These journalism students felt they needed to explain journalistic, critical source work and emphasize that they could not take sides in a conflict described in a story (interviewee, group 3); some had to explain the difference between a commercial webpage and a journalistic one (interviewees, group 2). However, because they were eager to collaborate and make efforts to understand one another, the students in group 3 used examples when explaining across disciplines (interviewees, group 3). Such discussions are part of learning about the other profession and sharing knowledge and culture in any interdisciplinary project and may help bridge the gap between two student groups (see also Weber \& Rall, 2012). 
Furthermore, the interviewees in group 3 emphasized that they all had ownership in the final result. This team began with brainstorming the journalistic idea in an interdisciplinary group. In group 4, where the members of the group listened to one another and shared knowledge, one student stated that the responsibility for the user was, in his opinion, divided evenly between journalists and technology developers (interviewee, group 4). In these two groups (3 and 4), the students benefited from mutual knowledge sharing. Due to collaboration, both groups of students acknowledged improved learning outcomes and gained awareness of the other field of practice (see also Angus \& Doherty 2015; Hultén \& Edwardsson, 2017; Kavanagh \& Cokley, 2011).

While they did not cross over or extend their professional boundaries, the students in the other two groups ( 1 and 2 ) did report learning from this practice-based project and gaining an improved awareness of the other students' knowledge. However, the computer science students in group 2 claimed to have learnt more from the experience of collaborating with other programmers, not from interacting with journalism students (interviewee, group 2). As mentioned above, group 1 operated under a client model, with the journalists having strong opinions about content and design, and the computer science students building the website accordingly. When the journalism students' requests were too difficult to implement, the computer science students explained why and focused on alternative solutions. In this process of translating abstract aims into action, they faced challenges by using the strategy of 'everybody was heard, everybody got to speak' (interviewee, group 1).

When placing experiences from the four groups along the axes of Collins and colleagues (2007), we found that group 3 and 4 can both be described as fractionated: they were both heterogeneous and collaborative groups where portions of the professional culture and language were exchanged in order to reach a common goal (Collins et al., 2007). As for the top-down authority structure of group 1, it resembles a subversive trading zone. However, there was obviously no cultural exchange, which characterizes an enforced trading zone that is heterogeneous and coercive. Group 2 is also best described as enforced.

\section{User perspectives}

We identified three different approaches with respect to the students' perspectives on user needs: 1) User orientation was given little consideration in the project, 2) user orientation was given some consideration or 3) user orientation was given considerable consideration, but mainly with regard to the dimensions of functionality and format. Among the journalists, the first experience was salient in our material. We found that the user perspective was 'totally forgotten, so it did not influence our work a lot' (journalist, questionnaire 2), 'did not affect us very much' (journalist, questionnaire 2) and 'was put on the backburner' (technology developer, questionnaire 2).

By comparison, the second experience was expressed as an ambition to 'reach as many as possible' (journalist, questionnaire 2) and 'working with users' needs in the back of our head' (technology developer, questionnaire 2). However, in line with Kavanagh and Cokley's (2011) remark on students' resistance in this perspective, more detailed reflections on target groups, user interest and user needs did not emerge.

In contrast, respondents who confirmed that user orientation did play an important role in the project focused on universal design dimensions and functionalities. User 
orientation 'affected our work in the sense that we had to consider users and to do additional work, like subtitling on video material and written text of audio files' (technology developer, questionnaire 2). In the interviews, the computer science students said that they see users as people with specific needs. During the process, they worked to achieve accessibility for everyone on the websites they were creating. By concentrating on design and functionality, their aim was easy navigation on the website, as with the use of skip navigation, and text quality (see also Aizpurua et al., 2016). They employed fonts, colour contrast and text sizes that were accessible and designed to be universal. They also used sound clips for people with vision impairments, and all videos included captions for those with hearing difficulties.

When it came to user perspectives, we found that the two student groups had notably different vocational approaches and acted as heterogeneous groups. Like the questionnaire, the interviews showed that users as targeted groups, interests and needs were not a concern for the journalists. When thinking about the user, they reflected along the lines of communication, of keeping the user engaged and, as Nordli (2016) writes, of improving the readability and comprehension of the texts. One journalist pointed out that the user is the one to whom they sought to communicate, adding that, to keep the user in a story, it has to be written so as to capture the reader's interest right from the start (interviewee, group 3).

Another journalism student expressed it like this: 'Our role is to make a story using understandable language and that the visual aspect with pictures and movies communicate in an effective and understandable way [...] so it becomes a complete story' (interviewee, group 2). This statement is in line with other journalists' opinions and Nordli's results (2016). Apart from that, they talked about clean design and interactivity, which gives the user options to choose and act. The journalism students agreed with the media industry that the use of interactivity and multimedia are some ways in which digitalized journalism could stimulate and engage users (see Peters, 2016). Together, the data results in this section provide multiple insights into the students' perspectives on user needs (RQ3).

\section{Conclusions}

One of the main questions for journalism today is the extent to which it can manage and capitalize on technological advances to remain relevant and fulfil its democratic roles. Building bridges between journalism and computer science is one way forward and a major challenge for journalism education. Journalism students trained for collaboration with computer science students are arguably better equipped to participate in and advance such collaboration in the work environment. The present small-scale study has demonstrated how a multitude of factors related to working in disciplinary cultures and power structures came into play when journalism students were asked to cooperate with computer science students. We found that the two sets of students represented heterogeneous cultures with distinct perceptions of their roles and responsibilities in the project.

Adding to research by Anderson and colleagues (2010), our data demonstrates how computer science students regarded their role as service providers catering to the needs of their journalism peers. Especially in team 1, the collaboration most closely resembled a client-provider relationship in which computer science students' responsibility was 
to bring the journalism students' feature stories to users through the design, form and functionalities of a webpage. This perception corresponded well with the journalism students' views of computer science students' roles and responsibilities. There was no indication that the computer science students wanted to expand their area of responsibility outside their own realm and into the journalistic field of gathering information and telling stories. Among the journalism students, we found tendencies towards a broader understanding of their role at the beginning of the project; some indicated responsibility for content production as well as design, form, and function. At the very least, that was the intention of some of the journalism students.

The in-between area of design was identified as the core of what Lewis and Usher $(2014,2016)$ refer to as a trading zone, where the interests of journalists and technology developers overlapped. Referring to Galison (1997), we may also call design a 'boundary object', which even though it 'means different things to the different parties [...] does not vitiate their separate projects' (Collins et al., 2007: 660). This was where their collaborative skills were put to the test and friction appeared in some groups (as observed in the workshops and reported upon in interviews). Design was also the area of mutual interest where the groups with the most extensive collaboration were able to draw on and integrate one another's heterogeneous competencies through knowledge sharing. In line with Collins and colleagues (2007: 660), we found that this collaboration involved 'fractions of cultures as the medium of interchange'. One possible explanation for students' different views of their roles and responsibilities may stem from the structured approach of the study, which differs from the more open-ended setting used by Angus and Doherty (2015).

As to the nature of the collaboration and the question of whether the students managed to find common ground, our findings are ambiguous. The students' engagement, expectations and priorities varied; they met challenges, but not all groups worked well together (see also Angus \& Doherty, 2015; Hultén \& Edwardsson, 2017; Kavanagh \& Cokley, 2011). Nevertheless, some groups established an early understanding of joint ownership and progressed with a strong feeling of working together; they ranked high on the collaborative dimension of their mutual trading zone, with both groups 3 and 4 fitting into the fractionated trading zone. Other groups kept within traditional boundaries; in these groups, the level of cooperation and sharing of knowledge was low. Both groups 1 and 2 were clearly heterogeneous and coercive, meaning that they fit into the enforced trading zone 'with almost no cultural interchange' (Collins et al., 2007: 660). Two factors were particularly decisive for the experience of coercion versus collaboration: the level of engagement of team members and the understanding of each other's competences. The groups that reached a truly common ground displayed strong dedication to the project and trust in the other team members' ability to get their jobs done. Our data suggests that collaboration and power dynamics were more a group-related experience than an experience of professional belonging, and personal dedication and the interests of each group member played roles in the collaborative process. In the words of Boden and Avram (2009), the groups that found common ground acted as knowledge brokers, and their personal inclinations helped to enact bridges of common language and understanding.

These findings expand previous research, which concluded that the literature on trading zones has little to say about individual-level influences (Lewis \& Usher, 2014). 
Our data shows that individual factors are clearly important for understanding the dynamics within trading zones. In addition, the concept of trading zones can be used to describe not only possibilities but also misunderstandings among interdisciplinary actors (Lewis \& Usher, 2016), and we observed both. However, apart from one situation, the students did not react defensively. Most students expressed an understanding of what the other profession had to bring to the table and how it would contribute to improving their joint assignment. That students with different training backgrounds respected one another's skills is in line with previous findings by Hultén and Edwardsson (2017) and Kavanagh and Cokley (2011). Some groups engaged in conversation about professional boundaries, gained new insights and were interested in further interdisciplinary work. This is a positive result that points to more opportunities for innovation and boundary negotiations in journalism.

The insights from this study provide guidance on the contingencies that may affect the process of working together in a technology-journalism project and the learning outcomes for students. Since most students are interested in design, that may be the starting point for further collaboration. In answering our main research question, we must reflect on our own beliefs in a student-centred, self-motivated project and admit that undergraduate students need well-defined structures to collaborate successfully (see also Kavanagh \& Cokley, 2011). As educators, we need to prepare students better before the next interdisciplinary project, both in how to engage with other professions and in how to think about audience analytics or target group thinking. Focus on design, better preparations and actively building on knowledge brokers may help establish a common language for realizing their aims in action (see Lewis \& Usher, 2016). In addition, the recommendation from Weber and Rall (2012) of a holistic, journalistic approach is worth considering in order to foster a more fruitful collaboration. This way, we can prepare the students on key concepts and start bridging the cultural gap between different disciplines. As Kavanagh and Cokley (2011) show, we observed that high expectations can create hurdles and expectations from the students, and the educators should therefore be addressed at an early stage. Furthermore, explicitly talking through possible challenges is recommendable.

The dimensions of heterogeneity versus homogeneity and coercion versus collaboration provide a framework for the evaluation and planning of future collaborative learning projects in journalism education. Working with these two dimensions, teachers are encouraged to experiment with different group constellations and tasks to better equip students to handle the collaborative challenges they will meet in the workplace. Furthermore, we encourage teachers to diligently apply practice-based learning challenges such as time pressure, restricted opportunity to choose collaborative partners and limited guidance on how to solve the task at hand. With this authenticity in our project, the lessons learned in the present study also may provide value for interdisciplinary projects in newsrooms.

While this study contributes to a more nuanced understanding of how journalism and computer science students collaborate in a team-based experiential learning project, it does have some limitations. First, the framing of the project (with limited time for interaction outside the workshops and no specific strategies to challenge the specialization of the two groups of students) may have affected the outcome of the project and made the students work in a more compartmentalized fashion than a more open-ended setting would have encouraged. Second, the study was a small project with only 33 students. 
Qualitative data from studies in other countries and courses may yield different results and normative implications. Future research is encouraged to expand the present research design to encompass other societal and educational contexts. As such, the present study represents a starting point for further research and practice.

\section{Funding}

This work was supported by the Norwegian Media Authority (RAM) for the project "En studie av profesjonsverdier og idealer i møtet mellom programmere, journalister og brukerbehov" [A study of professional values and ideals in the intersection between programmers, journalists and user needs] under grant no. 15/327-2/JEA15.

\section{Notes}

1. The computer science students came from the fields of applied information technology and software engineering.

2. While there is a growing body of literature on computing education for non-computer science majors (e.g., Pulimood et al., 2016; Wolz et al., 2011), this article leans more heavily towards the context of journalism. Consequently, it is beyond our scope to provide a thorough examination of computing education in non-computer science education.

\section{References}

Aizpurua, A., Harper, S. \& Vigo, M. (2016). Exploring the relationship between web accessibility and user experience. International Journal of Human-Computer Studies, 91: 13-23.

Anderson, K., Boyett, J., Courter, S. S., McGlamery, T., Nathans-Kelly, T. M. \& Nicometo, C. G. (2010). Understanding engineering work and identity: A cross-case analysis of engineers within six firms. Engineering Studies, 2(3): 153-174.

Angus, D. \& Doherty, S. (2015). Journalism meets interaction design: An interdisciplinary undergraduate teaching initiative. Journalism \& Mass Communication Educator, 70(1): 44-57.

Boden, A. \& Avram, G. (2009). Brigding knowledge distribution - The role of knowledge brokers in distributed software development teams. Paper presented at the CHASE'09/ICSE '09 Workshop, 2009, May 17, Vancouver, Canada.

Boyles, J. L. \& Meyer, E. (2016). Letting the data speak: Role perceptions of data journalists in fostering democratic conversation. Digital Journalism, 4(7): 944-954.

Collins, H., Evans, R.t. \& Gorman, M. (2007). Trading zones and interactional expertise. Studies in History and Philosophy of Science, 38: 657-666

Galison, P. (1997). Image and logic: A material culture of microphysics. Chicago: University of Chicago Press.

Gentikow, B. (2005). Hvordan utforsker man medieerfaringer? Kvalitativ metode [How to explore media experience? Qualitative method]. Kristiansand: IJ forlaget.

Gyori, B. \& Charles, M. (2018). Designing journalists: Teaching journalism students to think like web designers. Journalism \& Mass Communication Educator, 73(2): 200-217.

Han, G. K. \& Newell, J. (2014). Enhancing student learning in knowledge-based courses: Integrating team building in mass communication theory classes. Journalism \& Mass Communication Educator, 69(2): 180-196.

Hultén, G. \& Edwardsson, M. P. (2017). Storylab lessons. A collaborative project between courses in journalism and media technology. Nordicom Review, 39(1): 3-17.

Iwarsson, S. \& Ståhl, A. (2003). Accessibility, usability and universal design - Positioning and definition of concepts describing person-environment relationships. Disability \& Rehabilitation, 25(2): 57-66.

Kavanagh, L. \& Cokley, J. (2011). A learning collaboration between engineering and journalism undergraduate students prompts interdisciplinary behavior. Advances in Engineering Education, 2(3): 1-22.

Lees, J. (2001). Reflexive action research: Developing knowledge through practice. Counselling and Psychotherapy Research, 1(2): 132-138.

Lewis, S. C. \& Usher, N. (2014). Mapping the human-machine divide in journalism. In T. Witschge, C.-W. Anderson, D. Domingo \& A. Hermida (eds.), The SAGE handbook of digital journalism (pp. 341-353). Thousand Oaks: Sage. 
Lewis, S. C. \& Usher, N. (2016). Trading zones, boundary objects, and the pursuit of news innovation: A case study of journalists and programmers. Convergence: The International Journal of Research into New Media Technologies, 22(5): 543-560.

Lewis, S. C. \& Westlund, O. (2015). Actors, actants, audiences, and activities in cross-media news work: A matrix and a research agenda. Digital Journalism, 3(1): 19-37.

Newell, W. H. (2013). A theory of interdisciplinary studies. Issues in Integrative Studies, 19: 1-25.

Nordli, L. H. (2016). Identifying and overcoming organizational barriers in organizations to ensure universal design in practice: a case study of the Norwegian Broadcasting Corporation. Master's thesis, Oslo Metropolitan University.

Norman, D. A. (2001). The design of everyday things. Revised and expanded edition. New York: Basic Books.

Norman, D. A. \& Draper, S. W. (1986). User centered system design: New perspectives on human-computer interaction. Hillsdale: L. Erlbaum Associates.

O’Connell, T. \& Goldberg, L. (2011). Universal design in media. In K. H. Smith \& W. F. E. Preiser (eds.), Universal design handbook. New York: McGraw-Hill.

Pain, P., Chen, G. M. \& Campbell, C. P. (2016). Learning by doing: Teaching multimedia journalism through coverage of the 50th anniversary of Freedom Summer. Journalism \& Mass Communication Educator, 71(4): 400-412.

Parks, P. (2015). A collaborative approach to experiential learning in university newswriting and editing classes: A case study. Journalism \& Mass Communication Educator, 70(2): 125-140.

Peters, C. (2016). Spaces and places of news consumpion. In T. Witschge, C.-W. Anderson, D. Domingo \& A. Hermida (eds.), The SAGE handbook of digital journalism (pp. 354-369). Thousand Oaks: Sage.

Pulimood, S. M., Pearson, K. \& Bates, D. C. (2016). A study on the impact of multidisciplinary collaboration on computational thinking. In SIGSCE'16: Proceedings of the 47th ACM technical symposium on computing science education (pp. 30-35). New York: ACM.

Reese, S. D. (2016). The new geography of journalism research. Digital Journalism, 4(7): 816-826.

Weber, W. \& Rall, H. (2012). Data visualization in online journalism and its implications for the production process. Paper presented at the $16^{\text {th }}$ International Conference on Information Visualization, 2012, July $11-13$, at University of Montpellier.

Wolf, C. \& Schnauber, A. (2015). News consumption in the mobile era: The role of mobile devices and traditional journalism's content within the user's information repertoire. Digital Journalism, 3(5): 759-776.

Wolz, U., Cassel, L. B., Way, T. \& Pearson, K. (2011). Cooperative expertise for multidisciplinary computing. In SIGCSE '11: Proceedings of the 42nd ACM technical symposium on computer science education (pp. 329-334). New York: ACM.

Copyright: (C) 2019 The Author(s) and Nordicom. This is an open-access article distributed under the terms of the Creative Commons Attribution 4.0 International License (CC BY-NC-ND 4.0). 